\title{
Erratum to: Morphological and genetic differentiation of cultivated Undaria pinnatifida (Laminariales, Phaeophyta)
}

\author{
Kyosuke Niwa $^{1}$ - Atsushi Kobiyama ${ }^{2} \cdot$ Reiko Fuseya $^{3} \cdot$ Takashi Sakamoto $^{4}$
}

Published online: 8 March 2017

(C) Springer Science+Business Media Dordrecht 2017

\section{Erratum to: J Appl Phycol (2016) DOI 10.1007/s10811-016-1031-0}

The original version of this article unfortunately contained a mistake. Table 2 was incorrect. The correct Table 2 is now given below:

Table 2 Comparisons of size characteristics between cultivar 1 and cultivar 2 sporophytes of Undaria pinnatifida collected on January 8 and 25 and February 11, 2015

\begin{tabular}{|c|c|c|c|c|c|c|}
\hline & \multicolumn{2}{|l|}{ January 8, 2015} & \multicolumn{2}{|l|}{ January 25, 2015} & \multicolumn{2}{|c|}{ February 11, 2015} \\
\hline & Cultivar 1 & Cultivar 2 & Cultivar 1 & Cultivar 2 & Cultivar 1 & Cultivar 2 \\
\hline $\mathrm{TL}(\mathrm{cm})$ & $111.5 \pm 6.0 *$ & $100.8 \pm 6.7^{*}$ & $172.8 \pm 8.2 *$ & $149.1 \pm 12.7^{*}$ & $204.8 \pm 14.9$ & $199.8 \pm 15.5$ \\
\hline $\mathrm{BL}(\mathrm{cm})$ & $91.5 \pm 4.9^{*}$ & $83.9 \pm 4.9^{*}$ & $135.8 \pm 7.7^{*}$ & $123.4 \pm 10.3^{*}$ & $160.1 \pm 12.2$ & $167.2 \pm 13.2$ \\
\hline $\mathrm{BW}(\mathrm{cm})$ & $51.5 \pm 4.2^{*}$ & $34.0 \pm 3.5^{*}$ & $76.1 \pm 9.0^{*}$ & $53.2 \pm 3.4^{*}$ & $103.7 \pm 9.9 *$ & $54.9 \pm 3.8^{*}$ \\
\hline $\mathrm{DBL}(\mathrm{cm})$ & $24.8 \pm 3.2^{*}$ & $14.6 \pm 2.1 *$ & $36.4 \pm 3.2^{*}$ & $21.6 \pm 1.9^{*}$ & $49.7 \pm 6.6^{*}$ & $21.4 \pm 1.6^{*}$ \\
\hline UBW $(\mathrm{cm})$ & $6.9 \pm 1.3^{*}$ & $11.3 \pm 1.3^{*}$ & $10.5 \pm 1.3^{*}$ & $13.4 \pm 1.9^{*}$ & $11.2 \pm 1.0$ & $12.9 \pm 2.6$ \\
\hline $\mathrm{SL}(\mathrm{cm})$ & $20.0 \pm 2.2^{*}$ & $16.9 \pm 2.5^{*}$ & $37.0 \pm 2.8^{*}$ & $25.7 \pm 4.2^{*}$ & $44.6 \pm 3.8^{*}$ & $32.6 \pm 3.1 *$ \\
\hline $\mathrm{SW}(\mathrm{cm})$ & $1.4 \pm 0.1 *$ & $1.0 \pm 0.1 *$ & $2.4 \pm 0.2^{*}$ & $1.7 \pm 0.1 *$ & $2.9 \pm 0.2 *$ & $2.1 \pm 0.1 *$ \\
\hline $\mathrm{ST}(\mathrm{cm})$ & $0.4 \pm 0.1$ & $0.4 \pm 0.0$ & $0.5 \pm 0.0$ & $0.6 \pm 0.1$ & $0.7 \pm 0.1$ & $0.7 \pm 0.0$ \\
\hline $\mathrm{BT}(\mathrm{mm})$ & $0.136 \pm 0.024^{*}$ & $0.208 \pm 0.033 *$ & $0.289 \pm 0.030^{*}$ & $0.366 \pm 0.038^{*}$ & $0.374 \pm 0.061$ & $0.349 \pm 0.020$ \\
\hline TW (g) & $61.4 \pm 13.1$ & $61.7 \pm 10.4$ & $170.6 \pm 26.4$ & $172.3 \pm 22.8$ & $319.3 \pm 63.2$ & $286.8 \pm 32.5$ \\
\hline
\end{tabular}

Mean $\pm \mathrm{SD}(n=10)$. The asterisk indicates the significant difference between cultivar 1 and cultivar $2(p<0.05)$

$T L$ total length $(\mathrm{cm}), B L$ blade length $(\mathrm{cm}), B W$ blade width $(\mathrm{cm}), D B L$ longest divided blade length $(\mathrm{cm}), U B W$ undivided blade width $(\mathrm{cm}), S L$ stipe length (cm), $S W$ stipe width (cm), $S T$ stipe thickness (cm), $B T$ blade thickness (mm), $T W$ total wet weight $(\mathrm{g})$

The online version of the original article can be at http://dx.doi.org/10. 1007/s10811-016-1031-0.

Kyosuke Niwa

kyousuke_niwa@pref.hyogo.lg.jp

1 Fisheries Technology Institute, Hyogo Prefectural Technology Center for Agriculture, Forestry and Fisheries, Akashi, Hyogo 674-0093, Japan
2 School of Marine Biosciences, Kitasato University, Sagamihara, Kanagawa 252-0373, Japan

3 National Research Institute of Fisheries Engineering, Japan Fisheries Research and Education Agency, Kamisu, Ibaraki 314-0408, Japan

4 Department of Marine Biosciences, Tokyo University of Marine Science and Technology, Konan 4, Minato, Tokyo 108-8477, Japan 